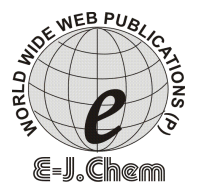

http://www.e-journals.net

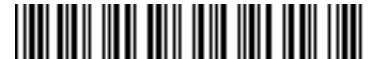

ISSN: 0973-4945; CODEN ECJHAO

E-Journal of Chemistry

Vol. 3, No.3, pp 122-133, July 2006

\title{
Higher Order Elastic Constants, Gruneisen Parameters and Lattice Thermal Expansion of Lithium Niobate
}

\author{
THRESIAMMA PHILIP*, C. S. MENON and K. INDULEKHA \\ School of Pure and Applied Physics, \\ Mahatma Gandhi University, \\ Kottayam, Kerala, India-686560
}

Received 24 January 2006; Accepted 14 April 2006

\begin{abstract}
The second and third-order elastic constants and pressure derivatives of second- order elastic constants of trigonal $\mathrm{LiNbO}_{3}$ (lithium niobate) have been obtained using the deformation theory. The strain energy density estimated using finite strain elasticity is compared with the strain dependent lattice energy density obtained from the elastic continuum model approximation. The second-order elastic constants and the non-vanishing third-order elastic constants along with the pressure derivatives of trigonal $\mathrm{LiNbO}_{3}$ are obtained in the present work. The second and third-order elastic constants are compared with available experimental values. The second-order elastic constant $C_{11}$ which corresponds to the elastic stiffness along the basal plane of the crystal is less than $C_{33}$ which corresponds to the elastic stiffness tensor component along the c-axis of the crystal. The pressure derivatives, $d C_{i j}^{\prime} / d p$ obtained in the present work, indicate that trigonal $\mathrm{LiNbO}_{3}$ is compressible. The higher order elastic constants are used to find the generalized Gruneisen parameters of the elastic waves propagating in different directions in $\mathrm{LiNbO}_{3}$. The Brugger gammas are evaluated and the low temperature limit of the Gruneisen gamma is obtained. The results are compared with available reported values.
\end{abstract}

Keywords: Elastic properties; Thermal expansion; Lattice dynamics 


\section{Introduction}

Lithium niobate $\left(\mathrm{LiNbO}_{3}\right)$ has received much attention in recent years because of its extensive applications in the field of device fabrications as well as material characterization. The stability of the phase over a wide range of temperature, and optical anisotropy explores its use as an efficient ferroelectric material ${ }^{1,2}$. It is also used in the field of electro- and elasto-optics because of its large electro-mechanical coupling coefficients ${ }^{3-5}$. An acoustical tone burst in the crystal makes it special in the field of acoustics and ultrasonics ${ }^{5-7}$. $\mathrm{LiNbO}_{3}$ finds its application also in the field of holographic imaging, optical waveguides and modern optical parametric oscillators $^{8-10}$. Its large spontaneous polarization and non linear optical activity make it favorite in the thermal, electrical and optical areas ${ }^{11,12}$. Its superior piezoelectric performance makes it a potential candidate for replacing quartz. The knowledge of higher order elastic constants is essential for the study of anharmonic properties of $\mathrm{LiNbO}_{3}$. Elastic constants also provide insight in to the nature of binding forces between the atoms since they are represented by the derivatives of the internal energy.

$\mathrm{LiNbO}_{3}$ exhibits a perovskite structure having two phases of trigonal symmetry with ten atoms per unit cell: a high symmetric paraelectric phase (space group $R \overline{3} c$ ) stable above $1480 \mathrm{~K}$ and a ferroelectric ground state(space group $R 3 c)^{13,14}$. In the high symmetry state $\mathrm{Li}^{+}$ions are located at the corners of the rhombohedral unit cell, $\mathrm{O}^{2-}$ atoms are at the face centers and the $\mathrm{Nb}^{5+}$ ion is occupying the body centre. The rhombohedral unit cell is defined by a lattice parameter of $5.492 \mathrm{~A}^{\circ}$ and an angle of $55^{\circ} 53^{\prime}$. The $\mathrm{LiNbO}_{3}$ structure can also be described as a hexagonal lattice with lattice dimension of $\mathrm{a}=5.151 \mathrm{~A}^{\circ}$ and $\mathrm{c}=13.876 \mathrm{~A}^{\circ}$.

The present objective is to study the vibrational anharmonicity of long wavelength acoustic modes of trigonal $\mathrm{LiNbO}_{3}$. Also here we make an attempt to calculate the complete set of second- and third-order elastic constants. Pressure derivatives of the second-order elastic constants and generalized Gruneisen parameters of elastic waves are also determined. Low temperature lattice thermal expansion of trigonal $\mathrm{LiNbO}_{3}$ is also obtained. The results are compared with those obtained by other workers ${ }^{15-22}$.

Cho et $a l^{20}$ determined all fourteen third order elastic constants of the crystal at room temperature from measured values of the velocity variation of small amplitude ultrasonic waves. Nakagawa et $\mathrm{al}^{21}$ have determined all fourteen elastic constants of the congruent crystal at room temperature by using the same method ${ }^{20}$ but they have determined the constants without correction. On the other hand Philip et $\mathrm{al}^{22}$ determined $C_{111}$ by measuring the amplitude of the second-harmonic wave of the longitudinal wave propagating along the $\mathrm{X}$-axis. In the case of determining $C_{111}$, this method does not need correction because the longitudinal wave propagating along the $\mathrm{X}$ - axis has no electromechanical coupling coefficient. The larger magnitudes of $C_{111}$ (as well as $C_{222}$ and $C_{333}$ ) appear to be more valid ${ }^{20-22}$.

Second and third order elastic constants.

Considering interactions up to third nearest neighbours in $\mathrm{LiNbO}_{3}$ the potential energy per unit cell ${ }^{23}$ is

$\phi=\phi_{0}+\sum_{I=1}^{3} \phi R(I)+\sum_{J=1}^{3} \phi R(J)$

The components of the interatomic vectors under a homogeneous deformation are given by 


$$
\begin{aligned}
& R_{i}^{\prime}(I)=R_{i}(I)+\sum_{j} \varepsilon_{i j} R_{j}(I) \text { and } \\
& R_{i}^{\prime}(J)=R_{i}(J)+\sum_{j} \varepsilon_{i j} R_{j}(J)+W_{i}
\end{aligned}
$$

where $\varepsilon_{i j}$ are the deformation parameters and are related to the macroscopic Lagrangian strains $\eta_{i j}$ by

$\eta_{i j}=\frac{1}{2}\left[\varepsilon_{i j}+\varepsilon_{j i}+\sum_{k} \varepsilon_{k i} \varepsilon_{k j}\right]$

$W_{i}$ are the components of the internal displacements of the lattice of particles of type $J$ relative to the lattice of particles of the type $I$ and are replaced by the relative internal displacements

$$
\overline{W_{i}}=W_{i}+\sum_{j} \varepsilon_{i j} W_{j}
$$

When the crystal is homogeneously deformed ${ }^{24}$ equation (2) becomes

$$
R_{i}^{\prime}(J)=R_{i}(J)+\sum_{j} \varepsilon_{i j} R_{j}(J)
$$

We expand the potential energy of the crystal in powers of the changes in the scalar products of the interatomic vectors ${ }^{25}$, and incorporate the two and three-body interactions in the expression for the potential energy. For instance, the two-body and three-body potentials among the first neighbour atoms ( $I$ atoms) are written, respectively, as $\phi^{(2)}=\frac{1}{2} \sum_{I}\left\{\frac{1}{2} \alpha_{1}\left[R^{\prime}(I) \cdot R^{\prime}(I)-R(I) \cdot R(I)\right]^{2}+\frac{1}{6} \xi_{1}\left[R^{\prime}(I) \cdot R^{\prime}(I)-R(I) \cdot R(I)\right]^{3}\right\}$

and

$$
\begin{aligned}
& \phi^{(3)}=\frac{1}{2} \sum_{I}\left[\frac{1}{2} \sigma_{1}\left\{\left[R^{\prime}(I) \cdot R^{\prime}\left(I^{\prime}\right)-R(I) \cdot R\left(I^{\prime}\right)\right]^{2}+\left[R^{\prime}(I) \cdot R^{\prime}\left(I^{\prime \prime}\right)-R(I) \cdot R\left(I^{\prime \prime}\right)\right]^{2}\right\}+\right. \\
& \left.\frac{1}{6} v_{1}\left\{\left[R^{\prime}(I) \cdot R^{\prime}\left(I^{\prime}\right)-R(I) \cdot R\left(I^{\prime}\right)\right]^{3}+\left[R^{\prime}(I) \cdot R^{\prime}\left(I^{\prime \prime}\right)-R(I) \cdot R\left(I^{\prime \prime}\right)\right]^{3}\right\}\right]
\end{aligned}
$$

Here $I^{\prime}$ and $I^{\prime \prime}$ are the neighbouring atoms lying on either side of a given atom $I$. The second-order parameters for the two-body interactions as well as the three-body interactions for the first neighbour atoms ( $I$ atoms) are written, respectively as $\alpha_{1}$ and $\sigma_{1}$. The thirdorder parameters for the two-body interactions as well as the three-body interactions for the first neighbour atoms ( $I$ atoms) are written, respectively as $\xi_{1}$ and $v_{1}$.

The strain energy derived from continuum model approximation ${ }^{24}$ is

$$
U=\frac{1}{2 !} \sum_{i j k l} C_{i j k l} \eta_{i j} \eta_{k l}+\frac{1}{3 !} \sum_{i j k l m n} C_{i j k l m n} \eta_{i j} \eta_{k l} \eta_{m n}+\ldots \ldots \ldots
$$

where $C_{i j k l}$ and $C_{i j k l m n}$ are the second and third order elastic constants in tensor form respectively. Comparing this with the lattice energy from equation (6) we get the expressions for the second-order and third-order elastic coefficients of trigonal $\mathrm{LiNbO}_{3}$. 
The second-order elastic coefficients are given in Voigt notation in equation (8).

$$
\begin{aligned}
& C_{11}=\left[57.81 \alpha_{1}+51.56 \alpha_{2}+658.84 \alpha_{3}+28.91 \sigma_{1}+25.78 \sigma_{2}\right] B \\
& C_{12}=\left[19.26 \alpha_{1}+17.19 \alpha_{2}+219.55 \alpha_{3}-9.64 \sigma_{1}-8.59 \sigma_{2}\right] B \\
& C_{13}=\left[0.70 \alpha_{1}+6.25 \alpha_{2}+9.72 \alpha_{3}-0.35 \sigma_{1}-0.31 \sigma_{2}\right] P^{2} B \\
& C_{14}=\left[0.60 \alpha_{1}+10.22 \alpha_{2}+12.02 \alpha_{3}-0.30 \sigma_{1}-5.11 \sigma_{2}\right] P B \\
& C_{33}=\left[0.46 \alpha_{1}+1.39 \alpha_{2}+0.93 \alpha_{3}+0.46 \sigma_{1}+1.39 \sigma_{2}\right] P^{4} B \\
& C_{44}=\left[0.70 \alpha_{1}+6.25 \alpha_{2}+9.72 \alpha_{3}+0.35 \sigma_{1}+0.31 \sigma_{2}\right] P^{2} B \\
& C_{66}=\left[19.27 \alpha_{1}+17.19 \alpha_{2}+219.61 \alpha_{3}+19.26 \sigma_{1}+17.19 \sigma_{2}\right] P B
\end{aligned}
$$

where $\quad B=10^{-2} \mathrm{a}^{4}$

Here ' $a$ ' is the lattice parameter and ' $P$ ' is the axial ratio c/a of the trigonal crystal $\mathrm{LiNbO}_{3}$. The second-order parameters characterizing the two-body interactions $\alpha_{1}, \alpha_{2}$ and $\alpha_{3}$ for the first, second and third neighbours and the three-body interactions $\sigma_{1}$ and $\sigma_{2}$ for the first and second neighbours are chosen to have an exact fitting of the experimental values of the second-order elastic constants of $\mathrm{LiNbO}_{3}$ measured by Takanaga et al ${ }^{15}$ and are given in Table 1. These values are used in Equation (8) to obtain the second-order elastic constants of $\mathrm{LiNbO}_{3}$. The values of second-order elastic constants of $\mathrm{LiNbO}_{3}$ thus obtained are given in Table 2 along with other reported values of Takanaga et $\mathrm{al}^{15}$, Ogi et $\mathrm{al}^{16}$, Kushibiki et al ${ }^{17}$, Kovacs et $\mathrm{al}^{18}$ and Damle ${ }^{19}$.

Table 1 Values of second-order potential parameters (in $\mathrm{G} \mathrm{Pa}$ ) of $\mathrm{LiNbO}_{3}$

\begin{tabular}{lc}
\hline Parameters & Values \\
\hline$\alpha_{1} B$ & -0.24 \\
$\alpha_{2} B$ & 1.24 \\
$\alpha_{3} B$ & 0.20 \\
$\sigma_{1} B$ & -1.57 \\
$\sigma_{2} B$ & 2.44
\end{tabular}

The third-order elastic coefficients are given in Voigt notation in equation (9).

$$
\begin{aligned}
& C_{111}=\left[129.91 \xi_{1}+250.95 \xi_{2}+3618.64 \xi_{3}+28.80 v_{1}-125.50 v_{2}\right] D \\
& C_{112}=\left[12.03 \xi_{1}+83.65 \xi_{2}+1065.51 \xi_{3}+6.02 v_{1}+41.80 v_{2}\right] D \\
& C_{113}=\left[0.44 \xi_{1}+28.20 \xi_{2}+42.52 \xi_{3}+0.21 v_{1}+14.10 v_{2}\right] P^{2} D \\
& C_{114}=\left[0.38 \xi_{1}+48.48 \xi_{2}+52.61 \xi_{3}+0.19 v_{1}+24.22 v_{2}\right] P D \\
& C_{123}=\left[0.15 \xi_{1}+9.40 \xi_{2}+14.17 \xi_{3}-0.07 v_{1}-4.70 v_{2}\right] P^{2} D \\
& C_{124}=\left[0.12 \xi_{1}+16.14 \xi_{2}+17.54 \xi_{3}-0.06 v_{1}-8.06 v_{2}\right] P D
\end{aligned}
$$




$$
\begin{aligned}
& C_{133}=\left[1.39 \xi_{1}+23.76 \xi_{2}+19.57 \xi_{3}-0.70 v_{1}-11.88 v_{2}\right] P^{2} D \\
& C_{134}=\left[0.17 \xi_{1}+5.51 \xi_{2}+3.34 \xi_{3}-0.08 v_{1}-2.75 v_{2}\right] P^{3} D \\
& C_{144}=\left[0.14 \xi_{1}+9.40 \xi_{2}+14.17 \xi_{3}-0.07 v_{1}-4.70 v_{2}\right] P^{2} D \\
& C_{155}=\left[0.44 \xi_{1}+28.20 \xi_{2}+42.51 \xi_{3}-0.07 v_{1}-4.70 v_{2}\right] P^{2} D \\
& C_{222}=\left[106.64 \xi_{1}+306.60 \xi_{2}+4187.41 \xi_{3}+52.43 v_{1}+69.68 v_{2}\right] D \\
& C_{333}=\left[0.13 \xi_{1}+0.64 \xi_{2}+0.26 \xi_{3}+0.13 v_{1}+0.64 v_{2}\right] P^{6} D \\
& C_{344}=\left[0.19 \xi_{1}+3.28 \xi_{2}+2.70 \xi_{3}+0.10 v_{1}+1.64 v_{2}\right] P^{4} D \\
& C_{444}=\left[-0.17 \xi_{1}-5.51 \xi_{2}-3.34 \xi_{3}-0.04 v_{1}-1.38 v_{2}\right] P^{3} D \\
& \text { where } \quad D=10^{-3} \mathrm{a}^{6}
\end{aligned}
$$

Table 2 Second-order elastic constants of $\mathrm{LiNbO}_{3}$ (in G Pa) along with the reported values

\begin{tabular}{lcccccc}
\hline \multirow{2}{*}{$C_{\mathrm{ij}}$} & \multirow{2}{*}{$\begin{array}{l}\text { Present } \\
\text { values }\end{array}$} & \multicolumn{5}{c}{ Reported Values` } \\
\cline { 3 - 7 } & 198.9 & 198.9 & 199.5 & 198.9 & 198.4 & 203.1 \\
\hline$C_{11}$ & 54.7 & 54.7 & 55.3 & 54.7 & 54.7 & 53.0 \\
$C_{12}$ & 67.3 & 67.3 & 67.7 & 68.0 & 65.1 & 74.2 \\
$C_{13}$ & 7.8 & 7.8 & 8.7 & 7.8 & 7.9 & 8.5 \\
$C_{14}$ & 233.7 & 233.7 & 235.2 & 234.2 & 227.9 & 241.3 \\
$C_{33}$ & 70.4 & 59.9 & 59.5 & 59.9 & 59.7 & 64.6 \\
$C_{44}$ & 72.1 & 72.1 & 72.1 & 72.1 & 71.8 & 75.1 \\
$C_{66}$ & & & & & & \\
\hline
\end{tabular}

The third-order potential parameters characterizing the two-body interactions $\xi_{1}, \xi_{2}$ and $\xi_{3}$ for the first, second and third neighbours and the three-body interactions $v_{1}$ and $v_{2}$ for the first and second neighbours are chosen to have an exact fitting of the experimental values of the third-order elastic constants of $\mathrm{LiNbO}_{3}$ reported by Cho et $\mathrm{al}^{20}$ in equation (9). The values of the third-order potential parameters thus calculated are given in Table 3 . The values from Table 3 have been used in equation (9) to obtain all the third-order elastic constants of $\mathrm{LiNbO}_{3}$. The values of third-order elastic constants thus obtained for $\mathrm{LiNbO}_{3}$ are collected in Table 4 along with the reported values of Cho et $a \mathrm{l}^{20}$ Nakagawa et a $\mathrm{l}^{21}$ and Philip et $a l^{22}$. 
Table 3 Values of third-order potential parameters (in $\mathrm{G} \mathrm{Pa}$ ) of $\mathrm{LiNbO}_{3}$

\begin{tabular}{lc}
\hline Parameters & Values \\
\hline$\xi_{1} D$ & -11.11 \\
$\xi_{2} D$ & -4.19 \\
$\xi_{3} D$ & 0.50 \\
$v_{1} D$ & 40.58 \\
$v_{2} D$ & 2.24
\end{tabular}

Pressure derivatives of the second order elastic constants.

The stress tensor $\tau_{i j}$ is defined by Murnaghan ${ }^{26}$ as

$\tau_{i j}=\frac{\rho}{\rho_{0}} \sum_{p q}\left[\frac{\delta x_{i}}{\delta a_{p}}\right]\left[\frac{\delta U}{\delta \delta_{p q}}\right]\left[\frac{\delta x_{j}}{\delta a_{q}}\right]$

where $\rho$ and $\rho_{0}$ are the densities $a_{j}$ and $x_{j}$ are the co-ordinates of material particles in the natural and deformed state respectively. $\eta_{i j}$ are the Lagrangian strain parameters and $U$ is the strain energy density.

Comparing this with the expression for stress

$\tau_{i j}=-p \delta_{i j}+\sum_{k l} C_{i j k l}^{\prime} \beta_{k l}$

$\beta_{k l}$ being the infinitesimal strain parameter and $p$ being the pressure, the expressions for the effective second-order elastic constants $C_{i j k l}^{\prime}$ can be obtained ${ }^{27}$ to the first-order in Lagrangian strains $\epsilon_{11}$ and $\epsilon_{33}$ as

$$
\begin{aligned}
& C_{11}^{\prime}=C_{11}+\epsilon_{11}\left[C_{111}+C_{112}+3 C_{11}+C_{12}\right]+\epsilon_{33}\left[C_{113}+C_{13}\right] \\
& C_{12}^{\prime}=C_{12}+\epsilon_{11}\left[C_{111}+2 C_{112}-C_{222}+2 C_{12}\right]+\epsilon_{33}\left[C_{123}\right] \\
& C_{13}^{\prime}=C_{13}+\epsilon_{11}\left[C_{113}+C_{123}+C_{13}\right]+\epsilon_{33}\left[C_{133}+C_{13}\right] \\
& C_{14}^{\prime}=C_{14}+\epsilon_{11}\left[C_{114}+C_{124}+2 C_{14}\right]+\epsilon_{33}\left[C_{134}\right] \\
& C_{33}^{\prime}=C_{33}+\epsilon_{11}\left[2 C_{133}+2 C_{13}\right]+\epsilon_{33}\left[C_{333}+3 C_{33}\right] \\
& C_{44}^{\prime}=C_{44}+\epsilon_{11}\left[C_{144}+C_{155}+C_{44}\right]+\epsilon_{33}\left[C_{344}+C_{44}\right] \\
& C_{66}^{\prime}=C_{66}+\epsilon_{11}\left[\frac{1}{2}\left[C_{222}-C_{112}\right]+2 C_{66}\right]+\epsilon_{33}\left[\frac{1}{2}\left[C_{113}-C_{123}\right]\right] \\
& \text { where } \in_{11}=\frac{\left[C_{13}-C_{33}\right] p}{\left[C_{11}+C_{12}\right] C_{33}-2 C_{13}^{2}} \text { and } \in_{33}=\frac{\left[2 C_{13}-C_{11}-C_{12}\right] p}{\left[C_{11}+C_{12}\right] C_{33}-2 C_{13}^{2}}
\end{aligned}
$$


The values of second-order and third-order elastic constants, given in Table 2 and Table 4 respectively, are substituted in equation (12) to get the pressure derivatives of the secondorder elastic constants of $\mathrm{LiNbO}_{3}$. The values thus obtained are given in Table 5

Table 4 Third order-elastic constants of $\mathrm{LiNbO}_{3}$ (in G Pa) along with the reported values

\begin{tabular}{|c|c|c|c|c|}
\hline \multirow{2}{*}{ Cijk } & \multirow{2}{*}{ Present work } & \multicolumn{3}{|c|}{ Reported values } \\
\hline & & Ref. [20] & Ref. [21] & Ref. [22] \\
\hline$C_{111}$ & -2120 & -2120 & -512 & -1610 \\
\hline$C_{112}$ & -97 & -530 & 454 & \\
\hline$C_{113}$ & -570 & -570 & 728 & \\
\hline$C_{114}$ & -361 & 200 & -410 & \\
\hline $\mathrm{C}_{123}$ & -301 & -250 & 79 & \\
\hline$C_{124}$ & -204 & 40 & 55 & \\
\hline$C_{133}$ & -749 & -780 & -34 & \\
\hline$C_{134}$ & -508 & 150 & -1 & \\
\hline$C_{144}$ & -300 & -300 & -37 & \\
\hline$C_{155}$ & -791 & -670 & -599 & \\
\hline$C_{222}$ & -2330 & -2330 & -599 & \\
\hline$C_{333}$ & -2960 & -2960 & -478 & \\
\hline$C_{344}$ & -774 & -680 & -540 & \\
\hline$C_{444}$ & 427 & -30 & -41 & \\
\hline
\end{tabular}

Table 5. Pressure derivatives of the second order elastic constants of $\mathrm{LiNbO}_{3}$

\begin{tabular}{cc}
\hline$d C_{i j}^{\prime} / d p$ & Present work \\
\hline$d C_{11}^{\prime} / d p$ & 6.36 \\
$d C_{12}^{\prime} / d p$ & 0.29 \\
$d C_{13}^{\prime} / d p$ & 4.26 \\
$d C_{14}^{\prime} / d p$ & 3.01 \\
$d C_{33}^{\prime} / d p$ & 9.81 \\
$d C_{44}^{\prime} / d p$ & 5.03 \\
$d C^{\prime} / d n$ & 3.50 \\
\hline
\end{tabular}


Generalized Gruneisen parameters of elastic waves and low temperature thermal expansion.

Uniaxial crystals are characterized by two principal linear expansion coefficients, $\alpha_{\|}$, parallel to the unique axis and $\alpha_{\perp}$, perpendicular to the unique axis. The behavior of these expansion coefficients at low temperature is governed by two generalized Gruneisen parameters (GP's) defined as

$$
\begin{aligned}
& \gamma_{j}^{\prime}(\theta, \phi)=-\frac{1}{v_{j}(\theta, \phi)} \frac{\partial v_{j}(\theta, \phi)}{\partial \varepsilon^{\prime}} \text { and } \\
& \gamma_{j}^{\prime \prime}(\theta, \phi)=-\frac{1}{v_{j}(\theta, \phi)} \frac{\partial v_{j}(\theta, \phi)}{\partial \varepsilon^{\prime \prime}}
\end{aligned}
$$

where $v_{j}(\theta, \phi)$, is the velocity of the elastic waves traveling in a direction $(\theta, \phi), j$, is the polarization index of the wave, $\theta$, is the angle the direction of wave propagation makes with the unique axis, $\phi$, is the azimuthal angle $\varepsilon^{\prime}$ is a uniform areal strain perpendicular to the unique axis and $\varepsilon^{\prime \prime}$ is a uniform longitudinal strain parallel to the unique axis. These generalized Gruneisen parameters can be calculated from the second and third-order elastic constants of a solid as shown by Ramji Rao and Srinivasan ${ }^{28}$. Using the second and thirdorder elastic constants the elastic wave velocities $v_{j}(\theta, \phi)$, the generalized Gruneisen parameters $\gamma_{j}^{\prime}(\theta, \phi)$ and $\gamma_{j}^{\prime \prime}(\theta, \phi)$ for different values of $\theta$ and $\phi$ at intervals of $5^{\circ}$ ranging from 0 to $90^{\circ}$ are calculated.

The linear thermal expansion coefficients of a uniaxial crystal are given by

$$
\begin{aligned}
& V \alpha_{\|}=\left[2 S_{13} \gamma^{\prime}(T)+S_{33} \gamma^{\prime \prime}(T)\right] C_{v}(T) \text { and } \\
& V \alpha_{\perp}=\left[\left(S_{11}+S_{12}\right) \gamma^{\prime}(T)+S_{13} \gamma^{\prime \prime}(T)\right] C_{v}(T)
\end{aligned}
$$

Here $V$ is the molar volume, the $S_{i j}$ are the elastic compliance coefficients, and $C_{v}(T)$ is the molar specific heat at temperature $T . \gamma^{\prime}(T)$ and $\gamma^{\prime \prime}(T)$ are the effective Gruneisen functions, being the weighted averages of the Gruneisen functions of all the normal modes of the crystal. At very low temperatures, the effective Gruneisen parameters are determined by the mode gammas of the elastic waves and $\gamma^{\prime}(T)$ and $\gamma^{\prime \prime}(T)$ attain limiting values, $\gamma_{0}^{\prime}$ and $\gamma_{0}^{\prime \prime}$. In terms of $v_{j}(\theta, \phi), \gamma_{j}^{\prime}(\theta, \phi)$ and $\gamma_{j}^{\prime \prime}(\theta, \phi)$, these limits are defined by

$$
\gamma_{0}^{\prime}=\frac{\sum_{j=1}^{3} \int v_{j}^{-3}(\theta, \phi) \gamma_{j}^{\prime}(\theta, \phi) d \Omega}{\sum_{j=1}^{3} \int v_{j}^{-3}(\theta, \phi) d \Omega} \quad \text { and }
$$


$\gamma_{0}^{\prime \prime}=\frac{\sum_{j=1}^{3} \int v_{j}^{-3}(\theta, \phi) \gamma_{j}^{\prime \prime}(\theta, \phi) d \Omega}{\sum_{j=1}^{3} \int v_{j}^{-3}(\theta, \phi) d \Omega}$

The integration is over the entire solid angle. We have obtained the values of $\gamma_{0}^{\prime}$ and $\gamma_{0}^{\prime \prime}$ by numerical integration over the solid angle. The integrals are evaluated by dividing $\theta$ and $\phi$ into intervals of $5^{\circ}$ and the values are obtained.

Brugger and Fritz ${ }^{29}$ have defined the functions

$\gamma_{\perp}^{B r}=V \alpha_{\perp} / C_{v} \chi_{\text {iso }} \quad$ and $\quad \gamma_{\|}^{B r}=V \alpha_{\|} / C_{v} \chi_{\text {iso }} \quad$ where $\chi_{\text {iso }}$ is the isothermal compressibility. Combining equation (14) and equation (15) the low temperature limits of the Brugger gammas are given by

$\gamma_{\perp}^{B r}(0)=\left[\left(S_{11}+S_{12}\right) \gamma_{0}^{\prime}+S_{13} \gamma_{0}^{\prime \prime}\right] / \chi_{\text {iso }}$

and

$\gamma_{\|}^{B r}(0)=\left[2 S_{13} \gamma_{0}^{\prime}+S_{33} \gamma_{0}^{\prime \prime}\right] / \chi_{\text {iso }}$

Here $S_{i j}$ are the elastic compliance coefficients and

$\chi_{\text {iso }}=2\left[S_{11}+S_{12}+S_{13}\right]+2 S_{13}+S_{33}$

Using the values of $\gamma_{0}^{\prime}$ and $\gamma_{0}^{\prime \prime}$ we get $\gamma_{\perp}^{B r}(0)$ and $\gamma_{\|}^{B r}(0)$.

Using these two values, the low temperature limit $\gamma_{L}$ can be calculated using the formula

$\gamma_{L}=2 \gamma_{\perp}^{B r}(0)+\gamma_{\|}^{B r}(0)$.

Figure 1 and Figure 2 show the variations of generalized Gruneisen parameters $\gamma_{j}^{\prime}$ and $\gamma_{j}^{\prime \prime}$ for the three elastic waves as a function of $\theta$. We have calculated the values of $\gamma_{0}^{\prime}$ and $\gamma_{0}^{\prime \prime}$ using Equation (15) as $\gamma_{0}^{\prime}=2.45$ and $\gamma_{0}^{\prime \prime}=2.15$. Using these values in Equation (16) the Brugger gammas are calculated and their values are $\gamma_{\perp}^{B r}(0)=0.95$ and $\gamma_{\|}^{B r}(0)=0.47$. The low temperature limit of the Gruneisen gamma is obtained as $\gamma_{L}=2.37$ for $\mathrm{LiNbO}_{3}$.

\section{Conclusion}

Trigonal $\mathrm{LiNbO}_{3}$ possesses seven second-order elastic constants. The values of second-order elastic constants of $\mathrm{LiNbO}_{3}$ obtained in the present work are collected in Table 2 along with other reported values of Takanaga et $\mathrm{al}^{15}$, Ogi et $a l^{16}$, Kushibiki et $a l^{17}$, Kovacs et $a l^{18}$ and Damle $^{19}$. The elastic constants $C_{i j}$ which corresponds to the wave propagation along the different axes of the crystal are in reasonable agreement with the available experimental values. Also it is found that $C_{33}$ is greater than $C_{11}$ which corresponds to the binding forces along the basal plane of the crystal. Therefore, in $\mathrm{LiNbO}_{3}$ the bonding between the atoms along the c-direction is stronger than that between the atoms along the a-b plane. Takanaga et $a l^{15}$ measured the elastic constants of $\mathrm{LiNbO}_{3}$ using line focus-beam acoustic microscopy and the values are found to be in good agreement except $C_{44}$ which differ from their value by 
17.5\%. Ogi et $a l^{16}$ have measured the elastic constants of $\mathrm{LiNbO}_{3}$ using acoustic spectroscopy and the values are found to be in good agreement except $C_{44}$ which differ from their value by $18.3 \%$. Kushibiki et $a l^{17}$ have also measured the elastic constants of $\mathrm{LiNbO}_{3}$ using line focus-beam acoustic microscopy and the values are found to be in good agreement except $C_{44}$ which differ their value by $17.5 \%$. The value of $C_{44}$ differ from the measurement of Kovacs et $a l^{18}$ by $17.9 \%$ and differ from the measurement of Damle ${ }^{19}$ by $9 \%$ respectively. The reported values of these constants by different workers ${ }^{15-19}$ differ considerably among themselves.

The third-order elastic constants evaluated in the present work are given in Table 4 along with the reported values of Cho et $a l^{20}$, Nakagawa et $a l^{21}$ and Philip $e$ t $a l^{22}$.The results obtained in the present work are of the same order with those of Cho et al ${ }^{20}$. All the thirdorder elastic constants of $\mathrm{LiNbO}_{3}$ are negative except $\mathrm{C}_{444}$. It is to be noted that there is only poor agreement between the reported experimental values of the third-order elastic constants 20, 21. This is because of the error in orienting the crystal during measurement. Also no theoretical values are reported so far. Hence we suggest a re-measurement which we expect to be in good agreement with the present work where we have considered two-body as well as three-body interactions up to the third neighbours. The third-order elastic constants deviate much in the experimental values ${ }^{20-22}$ as the measurements of the third-order elastic constants have high uncertainty.

The pressure derivatives of the second-order elastic constants of $\mathrm{LiNbO}_{3}$ obtained in the present work are given in Table 5. The pressure derivatives $d C_{i j}^{\prime} / d p$ obtained in the present work indicate that trigonal $\mathrm{LiNbO}_{3}$ is compressible.

The mode Gruneisen parameters of $\mathrm{LiNbO}_{3}$ for different acoustic wave propagation directions are calculated. This data give evidence for thermal expansion anisotropy of the material for various acoustic modes. $\gamma_{j}^{\prime \prime}$, which corresponds to the change in frequencies due to a uniform longitudinal strain along the c-axis of $\mathrm{LiNbO}_{3}$, is found to be more anisotropic than $\gamma_{j}^{\prime}$, which refers to change in frequencies due to a uniform areal strain perpendicular to the c-axis. Hence the vibrational anharmonicity along the c-axis is more pronounced than that along the a-b plane in $\mathrm{LiNbO}_{3}$. The variations of $\gamma_{j}^{\prime}$ and $\gamma_{j}^{\prime \prime}$ with $\theta$ are shown in Figure 1 and Figure 2. The anisotropy in all the graphs of $\gamma_{j}^{\prime}$ and $\gamma_{j}^{\prime \prime}$ vs. $\theta$ accounts for the pronounced anharmonicity of the solid in certain specific directions. The average Gruneisen functions are $\gamma_{\perp}^{B r}(0)=0.95$ and $\gamma_{\|}^{B r}(0)=0.47$. This suggests that the anisotropy in the thermal expansion along the c-axis is more pronounced than that along the a-b plane.

The low temperature limit of the lattice thermal expansion $\gamma_{L}$ is calculated from mode Gruneisen gammas and is found to have a value $\gamma_{L}=2.37$. The low temperature limit $\gamma_{L}$ is positive and hence we expect the volume lattice thermal expansion to be positive down to $0 \mathrm{~K}$ for $\mathrm{LiNbO}_{3}$. 


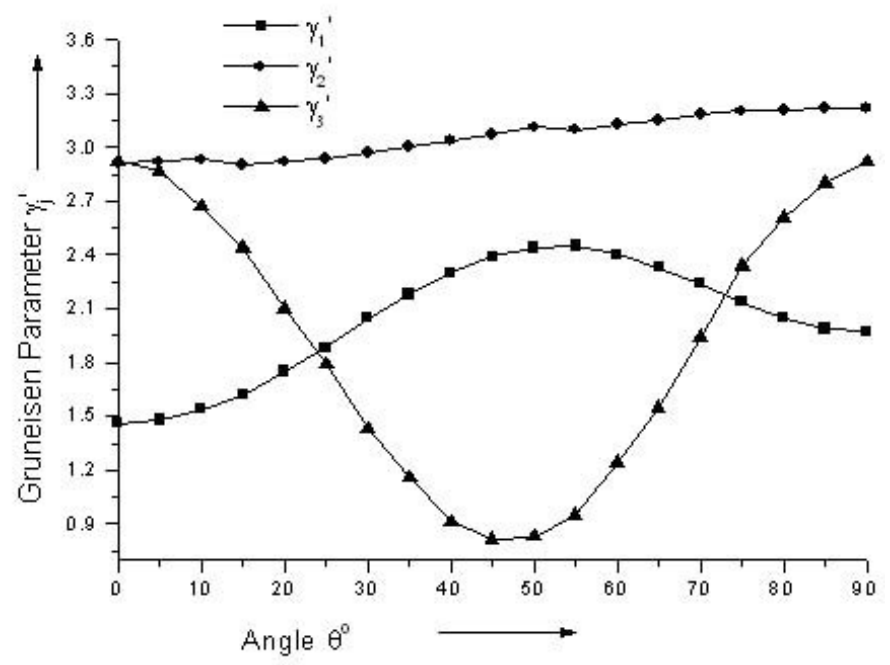

Figure 1 Variation of the generalized GP's $\gamma_{j}^{\prime}$ with angle $\theta$ for the different acoustic waves

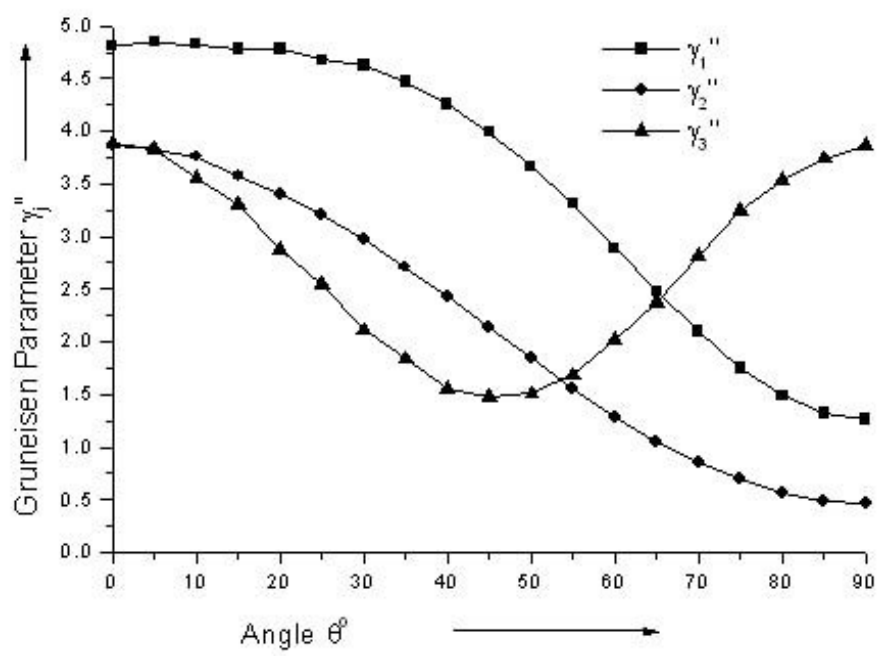

Figure 2 Variation of the generalized GP's $\gamma_{j}^{\prime \prime}$ with angle $\theta$ for the different acoustic waves

\section{References}

1. Jackson R A and Valerio M E G J. Phys. Condens. Matter 2005, 17, 837.

2. Scrymgeour D A, Gopalan V, Itagi A, Saxena A and Swart P J Phys. Rev. B 2005, 71, 184110.

3. Jazbinsek M and Zgonik M J. Appl. Phys. B 2002, 74, 407.

4. Kakio S and Nakagawa Y J. Appl. Phys. 1995, 34, 2917. 
5. Beyer O, Maxein D, Buse K, Sturman B, Hsieh H T and Psaltis D Phys. Rev. E 2005, 71, 056603.

6. McPherson M S, Ostrovskii I and Breazeale M A Phys. Rev. Lett. 2002, 89, 115506

7. Kushibiki J, Arakawa M and Okabe R IEEE Trans. Ultrason. Ferroelect. Freq. Contr. 2002, 49, 827.

8. Zhao J, Wang J G, Liu G D, He Q S, Wu M X and Jin G F Chin. Phys. Lett. 2003, 20, 377 .

9. Zhang P, Yang D X, Zhao J L, Su K, Zhou J B, Li B L and Yang D S Chin. Phys. Lett. 2004, 21, 1558.

10. Tillman K A, Maier R R J, Reid D T and McNaghten E D J. Opt. A: Pure Appl. Opt. 2005, 7, S408.

11. Singh, Saodekar P V and Bhoga S S Mater. Sci. 1998, 21, 469.

12. Chaib H, Otto T and Eng M Phys. Rev. B 2003, 67, 174109.

13. Veithen M and Ghosez P Phy. Rev. B 2002, 65, 214302.

14. Boysen H and Altorfer F Acta Crystallogr., Sect. B: Struct.Sci. B 1994, 50, 405.

15. Takanaga I and Kushibiki J IEEE Trans. Ultrason., Ferroelect., Freq. Contr. 2002, 49, 893.

16. Ogi H, Kawasaki Y, Hirao M and Ledbetter H J. Appl. Phys. 2002, 92, 20451.

17. Kushibiki J, Takanaga I, Arakawa M and Sannomiya T IEEE Trans. Ultrason., Ferroelect., Freq. Contr. 1999, 46, 1315.

18. Kovacs G , Anhorn M, Engan H E, Visintini G and Ruppel C C W, Proc. IEEE Ultrason. Symp.; Honolulu, Hawaii, 1990, 435.

19. Damle R V J. Appl. Phys. D 1992, 25, 1091.

20. Cho Y and Yamanouchi K J. Appl. Phys. 1987, 61, 875.

21. Nakagawa Y, Yamanouchi K and K Shibayama K J. Appl. Phys. 1973, 44, 3969.

22. Philip J and Breazeale M A IEEE US Symp. Proc. 1982, 2, 1022.

23. Mathew V M, Menon C S and Jayachandran K P J. Meterials Science 2002, 37, 5237.

24. Born M and Huang K, Dynamical Theory of Crystal Lattice; Oxford, London, 1954.

25. Keating P N Phys. Rev. 1966, 145, 637; 1966, 149, 674.

26. Murnaghan F D Finite Deformation of an Elastic Solid; Wiley, New York, 1951.

27. Ramji Rao R and Padmaja A J. Appl. Phys. 1987, 62, 440.

28. Ramji Rao R and Srinivasan R Phys. Status Solidi. 1968, 29, 865.

29. Brugger K and Fritz T C Phys. Rev. A 1967, 157, 524. 


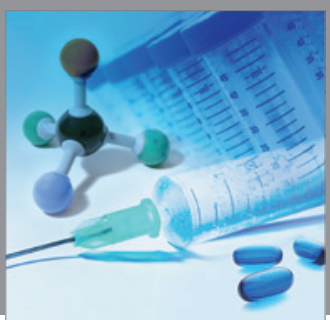

International Journal of

Medicinal Chemistry

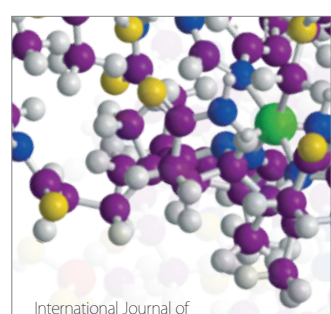

Carbohydrate Chemistry

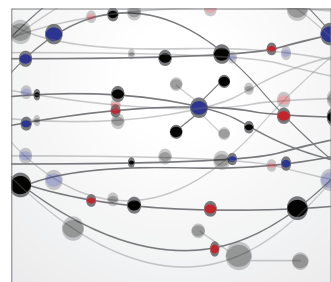

The Scientific World Journal
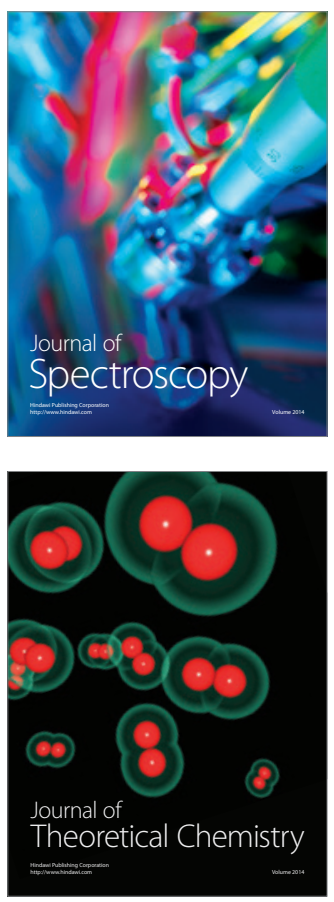
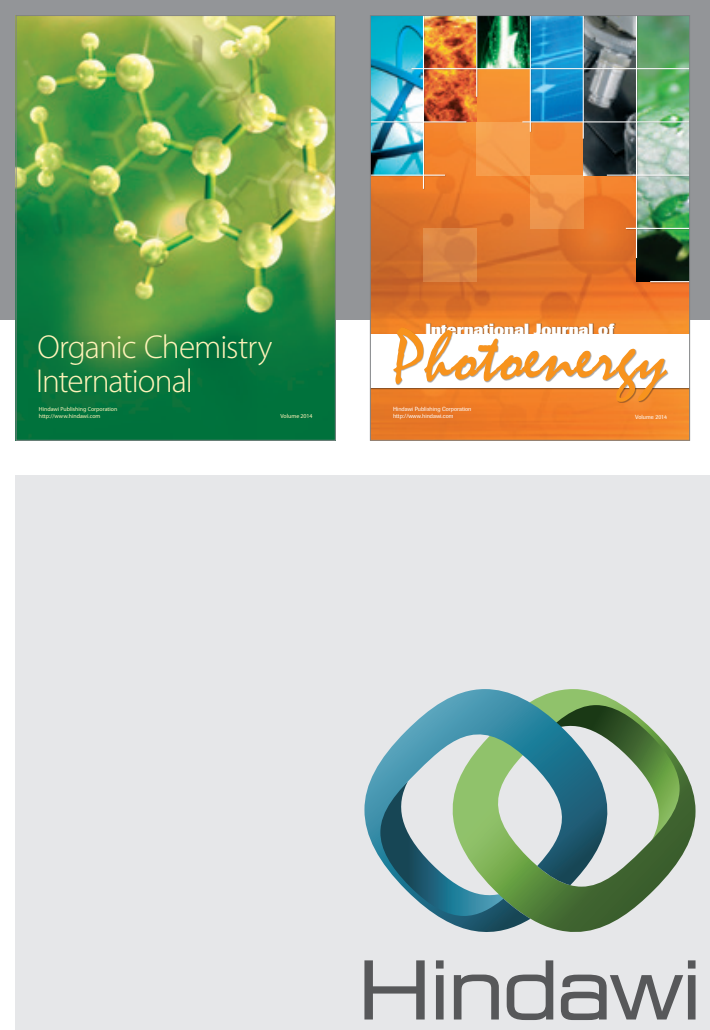

Submit your manuscripts at

http://www.hindawi.com
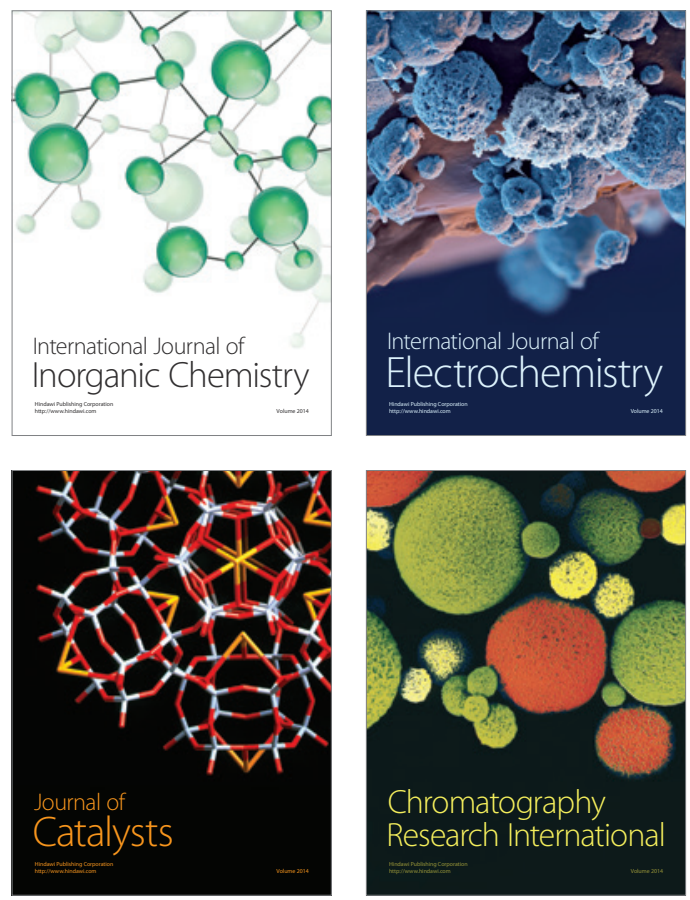
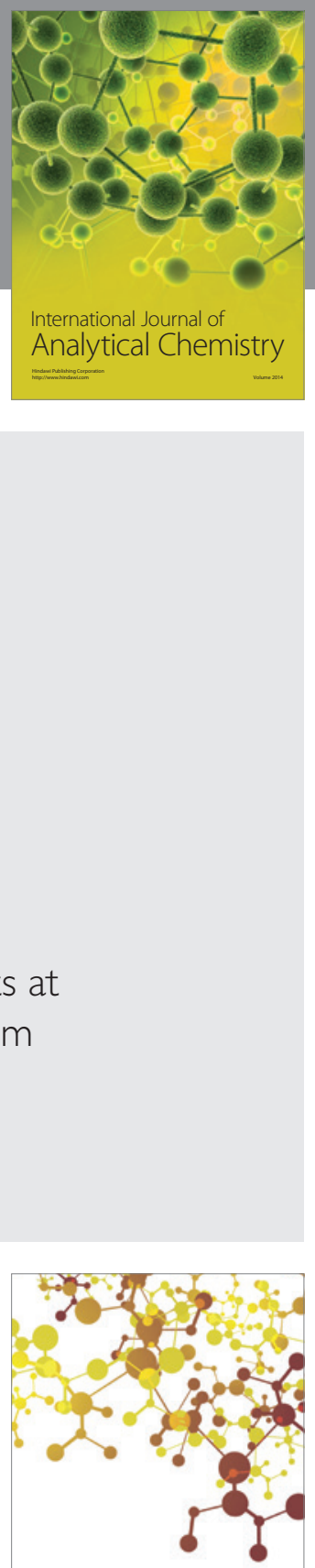

Journal of

Applied Chemistry
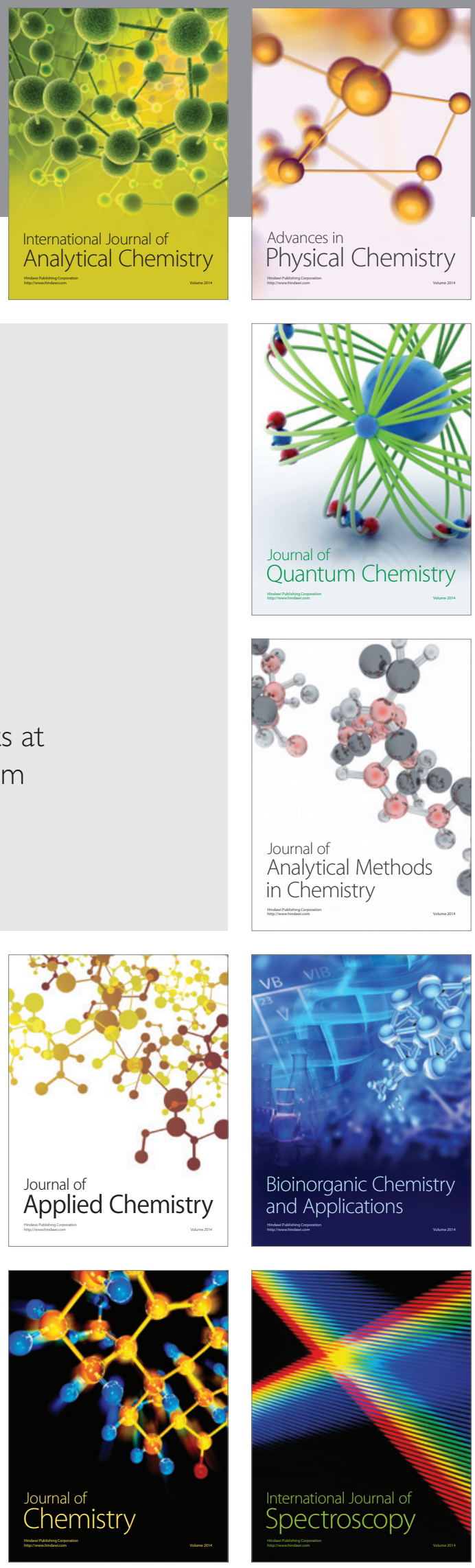